\title{
Uniform FDG-PET guided GRAdient Dose prEscription to reduce late Radiation Toxicity (UPGRADE-RT): study protocol for a randomized clinical trial with dose reduction to the elective neck in head and neck squamous cell carcinoma
}

Sven van den Bosch" ${ }^{*}$, Tim Dijkema ${ }^{1}$, Martina C. Kunze-Busch', Chris H. J. Terhaard ${ }^{2}$, Cornelis P. J. Raaijmakers ${ }^{2}$, Patricia A. H. Doornaert ${ }^{2}$, Frank J. P. Hoebers ${ }^{3}$, Marije R. Vergeer ${ }^{4}$, Bas Kreike ${ }^{5}$, Oda B. Wijers ${ }^{6}$, Wim J. G. Oyen ${ }^{7,8}$ and Johannes H. A. M. Kaanders ${ }^{1}$

\begin{abstract}
Background: In definitive radiation therapy for head and neck cancer, clinically uninvolved cervical lymph nodes are irradiated with a so-called 'elective dose' in order to achieve control of clinically occult metastases. As a consequence of high-resolution diagnostic imaging, occult tumor volume has significantly decreased in the last decades. Since the elective dose is dependent on occult tumor volume, the currently used elective dose may be higher than necessary.

Because bilateral irradiation of the neck contributes to dysphagia, xerostomia and hypothyroidism in a dose dependent way, dose de-escalation to these regions can open a window of opportunity to reduce toxicity and improve quality of life after treatment.

Methods: UPGRADE-RT is a multicenter, phase III, single-blinded, randomized controlled trial.

Patients to be treated with definitive radiation therapy for a newly diagnosed stage $T_{2-4} \mathrm{~N}_{0-2} \mathrm{M}_{0}$ squamous cell carcinoma of the oropharynx, hypopharynx or larynx are eligible. Exclusion criteria are recurrent disease, oncologic surgery to the head and neck area, concomitant chemotherapy or epidermal growth factor receptor inhibitors. In total, 300 patients will be randomized in a 2:1 ratio to a treatment arm with or without de-escalation of the elective radiation dose and introduction of an intermediate dose-level for selected lymph nodes. Radiation therapy planning FDG-PET/CT-scans will be acquired to guide risk assessment of borderline-sized cervical nodes that can be treated with the intermediate dose level.

Treatment will be given with intensity-modulated radiation therapy or volumetric arc therapy with simultaneousintegrated boost using an accelerated fractionation schedule, 33 fractions in 5 weeks. The primary endpoint is 'normalcy of diet' at 1 year after treatment (toxicity). The secondary endpoint is the actuarial rate of recurrence in electively irradiated lymph nodes at 2 years after treatment (safety).

(Continued on next page)
\end{abstract}

\footnotetext{
* Correspondence: sven.vandenbosch@radboudumc.nl

'Department of radiation oncology, Radboud University Medical Center,

huispost 874, P.O. Box 9101, Nijmegen 6500, HB, The Netherlands

Full list of author information is available at the end of the article
} 


\begin{abstract}
(Continued from previous page)
Discussion: The objective of the UPGRADE-RT trial is to investigate whether de-escalation of elective radiation dose and the introduction of an intermediate dose-level for borderline sized lymph nodes in the treatment of head and neck cancer will result in less radiation sequelae and improved quality of life after treatment without compromising the recurrence rate in the electively treated neck.
\end{abstract}

Trial registration: ClinicalTrials.gov Identifier: NCT02442375.

Keywords: Head and neck cancer, Squamous cell carcinoma, Accelerated radiation therapy, Dose reduction, Dose de-escalation, Elective nodes, FDG-PET, Euality of life

\section{Background}

In definitive radiation therapy for head and neck cancer, generally two dose-levels are delivered.

A high dose, the so-called "boost dose" to eradicate macroscopic tumor and a lower dose, the so-called "elective dose" to achieve control of clinically occult metastases in cervical lymph nodes. The current equivalent dose in 2 Gy fractions (EQD2) prescribed to the elective volume is $45-50 \mathrm{~Gy}$ and is based on literature from the nineteen-fifties [1]. At that time, assessment of the neck only consisted of physical examination due to the lack of sufficiently sensitive diagnostic imaging of lymph nodes.

Today, ultra-sound (US) with fine needle aspirated cytology (FNAC), computed tomography (CT) and magnetic resonance imaging (MRI) provide high resolution anatomical detail and have a high sensitivity and specificity in the detection of cervical lymph node metastases in head and neck cancer [2], even in a neck without palpable lymph nodes [3].

As a consequence of the implementation of highresolution diagnostic imaging techniques, tumor deposits measuring only a few millimeters are now detected and added to the boost volume. It is therefore plausible that nowadays occult tumor volume in radiologically uninvolved lymph nodes is much smaller than in the era before the implementation of these imaging techniques. However, the radiation therapy dose prescription practice for elective nodal regions has not changed over the years.

Since the dose required to control subclinical disease is dependent on occult tumor volume $[4,5]$, it would make sense to refine the traditional binary dose prescription to a more gradual one that is proportional to tumor volume. For this purpose, molecular imaging using fluorodeoxyglucose positron emission tomography (FDGPET) can improve the accuracy of current diagnostic imaging assuming that FDG-uptake represents tumor cell density, or at least is a good surrogate for this [6].

The potential value of FDG-PET imaging in the management of cervical lymph nodes lies in the decisionmaking process whether borderline-sized nodes should be treated with a boost or elective dose [7]. It is even conceivable that borderline-sized nodes having mild FDG-uptake may not require the maximum dose since they are likely to contain no or only a small tumor volume. An intermediate dose level may be sufficient for such nodes.

Because bilateral irradiation of cervical lymph node regions contributes to dysphagia, xerostomia and thyroid dysfunction in a dose dependent way $[8,9]$, dose reduction to these regions can open a window of opportunity to reduce toxicity and improve quality of life after treatment [10].

A treatment planning study performed at the Radboudumc showed that de-escalation of the elective dose as proposed in this study protocol, can reduce the dose to normal tissues such as the swallowing musculature, thyroid- and salivary glands (unpublished data). Normal tissue complication probability models from the available literature [11-15] showed that a relevant decrease in toxicity can be expected for xerostomia (absolute up to $14 \%$, relative risk up to $39 \%$ ), dysphagia (absolute up to $12 \%$, relative risk up to $67 \%$ ) and hypothyroidism (absolute up to $20 \%$, relative risk up to $50 \%$ ).

Given these considerations we believe that the traditional binary dose prescription in head and neck cancer is outdated. In this trial, a more gradual dose prescription will be used with de-escalation of the elective radiation dose and the introduction of an intermediate dose-level in the treatment of head and neck cancer. The aim is to investigate whether such a treatment will result in less radiation sequelae and improved quality of life after treatment (expressed as a normalcy of diet) without compromising the recurrence rate in electively irradiated lymph nodes.

\section{Methods/Design \\ Objectives}

To determine whether a more gradual dose prescription with de-escalation of the elective radiation dose and the introduction of an intermediate dose-level in the treatment of head and neck cancer will result in less radiation sequelae and improved quality of life after treatment (toxicity) without compromising the recurrence rate in electively irradiated lymph nodes (safety).

\section{Study design}

This is a multicenter, phase III, single-blinded, randomized controlled trial. 
Treatment allocation at randomization will be at a ratio of 2:1 in favor of the intervention arm. Randomization will be balanced for institution, tumor-site, T- and N-stage, and human papillomavirus status using minimization with a random element. Randomization and clinical trial data management is provided by the IKNL clinical research department.

A flow chart giving an overview of the study design is shown in Fig. 1.

\section{In- and exclusion criteria}

Adult patients having a new, pathologically proven squamous cell carcinoma located in the larynx, oropharynx or hypopharynx with stage $\mathrm{T}_{2-4} \mathrm{~N}_{0-2} \mathrm{M}_{0}$ are eligible for inclusion after written informed consent. Patients must be able to undergo accelerated radiation therapy and have a World Health Organization performance status of $0-2$.

Main exclusion criteria are concomitant chemotherapy or epidermal growth factor receptor inhibitors for this tumor, prior anticancer treatment to the head and neck area (surgery, chemotherapy or radiation therapy), previous malignancies or uncontrolled diabetes mellitus.

\section{Endpoints}

Primary endpoint (toxicity): 'normalcy of diet' at 1 year after treatment, measured using the performance status scale for patients with head and neck cancer (PSS-HN) [16].

Secondary endpoint (safety): actuarial rate of recurrence in electively irradiated lymph nodes at 2 years after treatment.

Other endpoints:

Acute toxicity (mucositis, dysphagia and skin reaction).

Late toxicity (with focus on xerostomia, dysphagia and hypothyroidism).
Quality of life (general-, xerostomia- and dysphagia related quality of life).

Recurrence (local, regional, loco-regional and distant). Survival (overall, disease specific and disease free).

\section{Sample size calculation}

This study was designed to detect a 10-point difference on the PSS-HN 'normalcy of diet' score at 12 months after radiation therapy with a power of $90 \%$ at a two-sided significance level of 0.05. An average 'normalcy of diet' score of 70 is expected after standard treatment. To achieve this significance level with an unequal randomization ratio (2:1), a total of 300 patients needs to be included.

The current rate of recurrence in electively irradiated lymph nodes was estimated to be $5 \%$ at 2 years after treatment [17]. An equal rate of recurrence is expected in the intervention arm, despite elective dose deescalation. A recurrence rate of $\geq 10 \%$ will be considered clinically relevant and unacceptable. This difference can be detected with the number of patients planned for the primary outcome of the study and a one-sided $\alpha=0.10$.

The total duration of this trial is estimated to be 6 years ( 4 years accrual, 2 years follow-up).

\section{Pre-treatment evaluation}

Pre-treatment evaluation will include physical examination and flexible endoscopy of the upper aerodigestive tract, biopsy of the tumor, MRI and/or CT-scan of the head and neck area, and US of the neck including FNAC of cervical lymph nodes. All patients are evaluated by a multidisciplinary head-and-neck oncology team.

\section{Radiation therapy planning FDG-PET/CT-scan}

In order to ensure that in the multicenter setting of this trial, the acquired quantitative data and standardized uptake

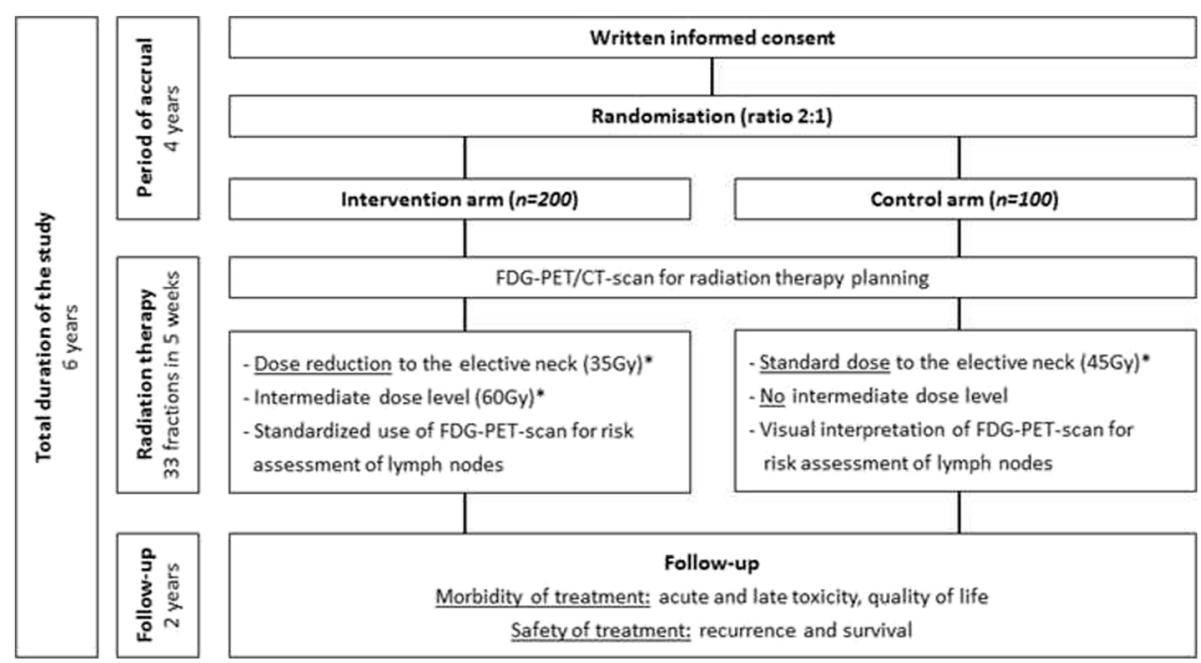

Fig. 1 Flow chart giving an overview of the study design. *the reported dose is the equivalent dose in 2 Gy fractions (EQD2) 
value (SUV) recoveries are interchangeable between study sites, all FDG-PET/CT-scans will be acquired on EARL accredited scanners (http://earl.eanm.org) following the European Association of Nuclear Medicine (EANM) procedure guidelines for tumor PET imaging v2.0 [18].

FDG-PET/CT-scans of the head and neck area will be acquired in radiation therapy treatment position using a custom-made thermoplastic head, neck and shoulders mask to immobilize the patient during radiation therapy and the scanning procedures. A diagnostic CT-scan using an intravenous contrast agent will be acquired in one session on the PET/CT-scanner for treatment planning.

\section{Standardized uptake ratio (SUR)}

In this trial, the SUV will be normalized by an internal image-derived standard, in order to minimize inter-study variability of FDG-uptake [18]. The cervical spinal cord will be used as the internal image-derived standard [19].

The SUR will be calculated using the following formula:

$\mathrm{SUR}_{\max }=\left(\mathrm{SUV}_{\text {max }}\right.$ in region of interest $/ \mathrm{SUV}_{\text {mean }}$ of internal standard ).

\section{Risk assessment of lymph nodes Intervention arm}

In the intervention arm, lymph nodes will be classified into three risk-levels and will be treated with a corresponding radiation dose. The FDG-PET-scan will guide risk assessment using standardized methods in order to minimize inter-institutional and inter-operator variations.

- High-risk (macroscopic tumor): comprises metastatic nodes that will be identified by (1) positive cytology or (2) necrosis on imaging or (3) $\mathrm{SUR}_{\max } \geq 2.0$.

- Intermediate-risk: comprises lymph nodes of borderline size having intermediate FDG-uptake and will be identified by (1) summed long- and short-axis diameter $\geq 17 \mathrm{~mm}$ and (2) $\mathrm{SUR}_{\max } \geq 1.5$ and $<2.0$ and (3) cannot be high-risk (e.g. positive cytology or necrosis on imaging). These criteria are based on an in-depth risk assessment on recurrence in 1166 electively irradiated nodes in 264 patients. Not overtly pathologic lymph nodes with a summed diameter $\geq 17 \mathrm{~mm}$ had an increased risk to recur after elective treatment (Hazard Ratio: 17.8, 95\%CI: 5.7-55, $p<0.001)$ [17].

- Low-risk (microscopic tumor): comprises elective lymph node regions defined in the protocol based on tumor site and stage and will be delineated according to published guidelines [20].

Retropharyngeal lymph nodes will be evaluated by traditional means as standardized FDG-PET-guided riskassessment is not applicable to these nodes.

\section{Control arm}

In the control arm, no areas of intermediate risk will be identified. Lymph nodes are either assigned to the highrisk (macroscopic) volume or low-risk (elective) volume by traditional means (positive cytology or necrosis on imaging or short-axis diameter $\geq 10 \mathrm{~mm}, \geq 11 \mathrm{~mm}$ for subdigastric nodes).

The FDG-PET-scan may be used in the decision making process by means of visual interpretation.

\section{Delineation and margins}

For the primary tumor, the gross target volume $\left(G_{T V}\right)$ will be delineated by traditional means using information from clinical examination and diagnostic imaging (CT and/or MRI) and will encompass all overtly macroscopic disease. In both treatment arms, a biological target volume $\left(\mathrm{BTV}_{\mathrm{p}}\right)$ of the primary tumor is created by means of adaptive threshold iso-contouring based on the FDGSUR maps [19]. Gross / high-risk lymph node metastases will be delineated separately $\left(G_{T V}\right)$.

A clinical target volume (CTV) is created to cover all routes of potential subclinical disease spread.

The GTV $\mathrm{V}_{\mathrm{p}}$ will be expanded by a 3D margin of $10 \mathrm{~mm}$ and the $\mathrm{GTV}_{\mathrm{n}}$ will be expanded by a 3D margin of $5 \mathrm{~mm}$ (10 $\mathrm{mm}$ in case of extra nodal disease on imaging) in order to create the $\mathrm{CTV}_{\text {high-risk. }}$ The $\mathrm{CTV}_{\text {high-risk }}$ will be adjusted for anatomical borders in which microscopic disease is unlikely to extend. No CTV expansion will be used for intermediate-risk lymph nodes as extra nodal disease is unlikely in these nodes.

To take patient set-up uncertainties into account, a planning target volume (PTV) will be created by extension of the CTV with a 3D margin of 3-5 mm (according to the participating centers protocols). Additionally, for the high-risk tumor volume an additional $\mathrm{PTV}_{\mathrm{BGTV} \text {-high-risk }}$ will be created by extension of the $\mathrm{BTV}_{\mathrm{p}}$ and $\mathrm{GTV}_{\mathrm{n}}$ with a $3 \mathrm{D}$ margin of 3$5 \mathrm{~mm}$. Differences in target volumes and dose prescription between the treatment arms are illustrated in Fig. 2 .

Organs at risk will be delineated according to published international consensus guidelines [21]. Standardized naming of target volumes and organs at risk will facilitate inter-institutional data analysis in this multicenter trial [22].

\section{Radiation therapy regimen}

Patients will be treated with accelerated external beam radiation therapy (EBRT) using volumetric modulated 

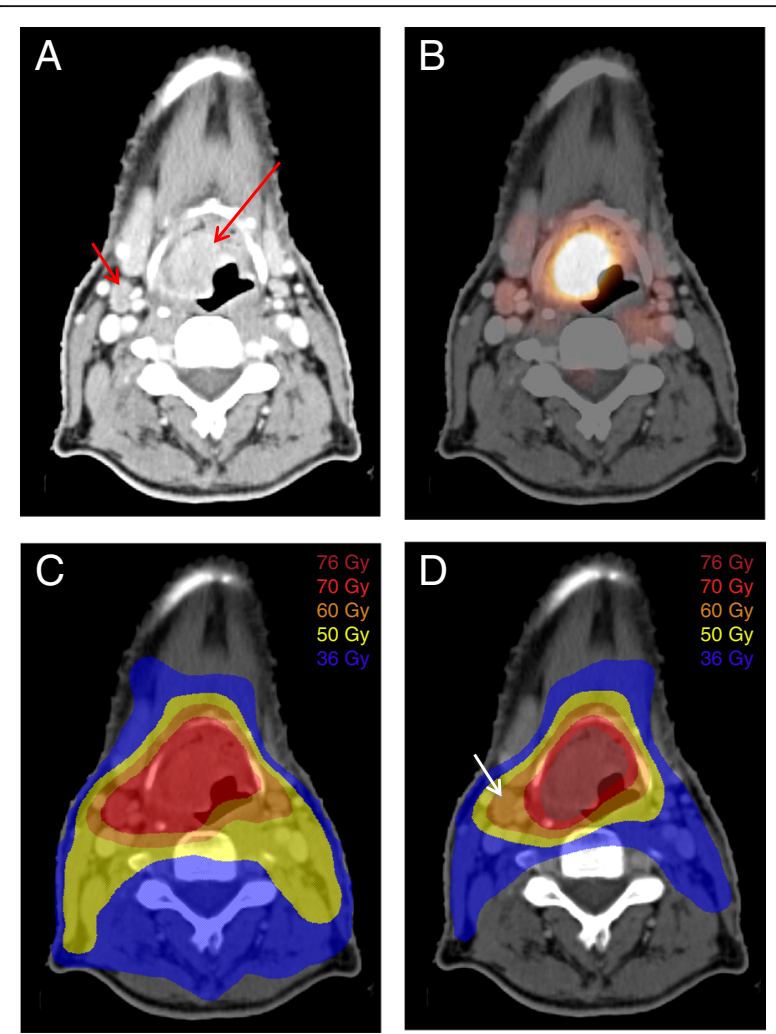

Fig. 2 Radiation therapy planning FDG-PET/CT-scan of a patient with an laryngeal squamous cell carcinoma (red arrow) with an intermediate risk lymph node in level 3 right (red arrow) $(\mathbf{a}+\mathbf{b})$. Comparison of dose planning conform this study protocol for the control-arm (c) and intervention-arm (d) shows the potential of FDG-PET guided gradient dose prescription with dose reduction to the elective neck in order to better spare organs at risk

arc therapy (VMAT) or intensity modulated radiation therapy (IMRT) with simultaneous integrated boost (SIB) techniques to deliver multiple dose levels. The total treatment consists of 33 fractions in an overall treatment time of 33 days ( 5 weeks). During the first 4 weeks of treatment, 6 fractions will be delivered in 5 consecutive days per week. In the last week of treatment, 9 fractions will be delivered (i.e. 4 days bid treatment). The interval between fractions will be at least $6 \mathrm{~h}$. According to the participating centers protocols, offline or online cone beam CT-scans will be made during treatment to verify correct positioning of the patient during irradiation.

Dose prescriptions for the treatment arms are shown in Table 1.

\section{Follow-up}

Acute toxicity will be evaluated weekly during treatment and every 2 weeks thereafter, until acute toxicity is completely healed. Acute toxicity will be scored using the common toxicity criteria v2.0 [23]. Standard oncologic
Table 1 Dose prescription

\begin{tabular}{llll}
\hline Target volume & \multicolumn{2}{l}{ Dose (fraction dose) (Gy) } & ${ }^{a} E Q D 2$ (Gy) \\
\cline { 2 - 3 } & Intervention-arm & Control-arm & \\
\hline PTV $_{\text {GBTV-high-risk }}$ & $66(2.00)$ & $66(2.00)$ & $\approx 73$ \\
PTV $_{\text {CTV-high-risk }}$ & $62(1.88)$ & $62(1.88)$ & $\approx 67$ \\
PTV $_{\text {intermediate-risk }}$ & $58(1.76)$ & - & $\approx 60$ \\
PTV $_{\text {low-risk }}$ & $42(1.27)$ & $48(1.45)$ & $\approx 35$ vs. 45
\end{tabular}

${ }^{a}$ The equivalent dose in 2 Gy fractions (EQD2) was calculated using the linearquadratic model using an $\alpha / \beta=10 \mathrm{~Gy}$ for tumor [34]. Differences in treatment time were taken into account by a correction of 0.6 Gy per day to compensate for tumor repopulation [35]

An accelerated fractionation schedule will be used, 33 fractions in 5 weeks (33 days)

follow-up visits are scheduled every 2 months during the 1st year and every 3 months during the 2nd year, after which study participation ends. However, oncologic follow-up will continue every 4 months during the 3rd year and twice annually until at least 5 years of followup. During oncologic follow-up visits, late toxicity, recurrence and survival will be evaluated. Late toxicity will be scored using the RTOG/EORTC late radiation morbidity scoring criteria [24].

Periodical study visits will be scheduled once before treatment and at 3, 6, 12 and 24 months after radiation therapy. Subjects will undergo assessment of the swallowing function, thyroid- and salivary glands function and quality of life questionnaires will be completed.

A schedule of study procedures is shown in Table 2.

Table 2 Schedule of study procedures

\begin{tabular}{|c|c|c|c|c|c|c|c|}
\hline \multirow[t]{2}{*}{ Procedure } & \multirow[t]{2}{*}{$\begin{array}{l}\text { Before } \\
\text { treatment }\end{array}$} & \multirow[t]{2}{*}{$\begin{array}{l}\text { During } \\
\text { treatment }\end{array}$} & \multicolumn{5}{|c|}{$\begin{array}{l}\text { Months after } \\
\text { treatment }\end{array}$} \\
\hline & & & 0 & 3 & 6 & 12 & 24 \\
\hline Planning FDG-PET/CT-scan & $x$ & & & & & & \\
\hline Acute toxicity (CTC v2.0) & & $x$ & $x$ & $x$ & & & \\
\hline Late toxicity (RTOG-EORTC) & & & & $x$ & $x$ & $x$ & $x$ \\
\hline $\begin{array}{l}\text { Assessment of thyroid function } \\
\text { (Blood analysis) }\end{array}$ & $x$ & & & & $x$ & $x$ & $x$ \\
\hline $\begin{array}{l}\text { Dysphagia related quality of life } \\
\text { (PSS-HN, SWAL-QOL) }\end{array}$ & $x$ & & & $x$ & $x$ & $x$ & $x$ \\
\hline $\begin{array}{l}\text { Assessment of swallowing } \\
\text { function } \\
\text { (Water swallowing test) }\end{array}$ & $x$ & & & $x$ & & $x$ & $x$ \\
\hline $\begin{array}{l}\text { Xerostomia related quality } \\
\text { of life } \\
(G R I X)\end{array}$ & $x$ & & & $x$ & $x$ & $x$ & $x$ \\
\hline $\begin{array}{l}\text { Assessment salivary gland } \\
\text { function } \\
\text { (sialometry, sialochemistry) }\end{array}$ & $x$ & & & $x$ & & $x$ & $x$ \\
\hline $\begin{array}{l}\text { General quality of life } \\
\text { (EORTC QLQ-C30, } \\
\text { EORTC H\&N35) }\end{array}$ & $x$ & & & $x$ & $x$ & $x$ & $x$ \\
\hline Assessment of recurrence & & & & $\begin{array}{l}\text { ich } \\
\text { sit }\end{array}$ & & & \\
\hline
\end{tabular}




\section{Assessment of recurrence}

If recurrence is suspected during follow-up, additional imaging by MRI, CT, PET or US with FNAC will be performed whatever is judged necessary by the attending physician. Examination under general anesthesia is performed if deemed necessary. All recurrences must be confirmed by cytology or histology.

Central evaluation will be done for all regional recurrences in order to determine if the recurrence occurred in an electively irradiated lymph node. The exact site of recurrence will be reconstructed by performing co-registration of the planning CT-scan with diagnostic imaging of the recurrence.

All recurrences in electively irradiated lymph nodes will be reported as a serious adverse event since this is the safety endpoint of this trial.

\section{Functional assessments}

- Salivary gland function will be evaluated in a part of the participating centers only. Stimulated parotid and submandibular salivary flow rates (sialometry) will be measured using techniques described previously [25, 26]. Samples of saliva collected with sialometry will be analyzed for its composition (sialochemistry).

- Swallowing function will be evaluated using the water swallowing test [27]. Additional functional performance will be evaluated by the Performance Status Scale for Head \& Neck Cancer Patients (PSSH-HN) [16].

- Thyroid gland function will be evaluated using standard blood analysis measuring the thyroid stimulating hormone and free thyroxin.

\section{Assessment of quality of life}

For evaluation of general quality of life, the EORTC QLQC30 and EORTC QLQ-H\&N35 questionnaires will be used $[28,29]$. Xerostomia related quality of life will be evaluated using the Groningen Radiation Therapy Induced Xerostomia questionnaire (GRIX) [30] and dysphagia related quality of life will be evaluated using the Swallowing Quality of Life Questionnaire (SWAL-QOL) [31].

\section{Statistical analysis}

All analyses concerning treatment effects will be done according to the intention to treat principle. The student's t-test will be used to compare 'normalcy of diet' scores at 1 year after treatment, the primary endpoint of this trial. A Kaplan-Meier estimate will be calculated for the actuarial rate of recurrence in electively irradiated lymph nodes at 2 years after treatment. Actuarial rates on recurrence and survival will be determined by the date of histopathological diagnosis. Differences between the treatment arms will be assessed using the log-rank test.

For each quality of life questionnaire, data will be included in the analysis if a patient filled in the questionnaire at least at start and at one time-point during the study. Differences in quality of life over time between the intervention- and control arm will be analyzed by using a linear mixed model for repeated measurements. Difference in quality of life scores $\geq 10$ points will be considered clinically relevant [32].

\section{Safety assurance}

After every 5 recurrences in electively irradiated lymph nodes, an interim analysis will be performed following the 'group sequential approach' comparing the recurrence rate in the two treatment arms [33]. The $p$-value for the log-rank test statistic will be compared to a nominal $\alpha$ of 0.042 at each interim analysis (i.e. critical value of 1.728) to ensure an overall one-sided $\alpha$ of 0.10 [33]. For this calculation, it is assumed that accrual of 300 patients takes 4 years and that the vast majority of recurrences will be detected within 24 months.

A safety committee will be installed to undertake interim review of the trial safety. The safety committee will consist of an independent statistician and two experienced radiation oncologists in the field of head and neck cancer and will recommend on (dis)continuation of the trial.

\section{Quality assurance}

In order to ensure quality and uniformity between centers, delineation, segmentation and treatment planning guidelines are described in detail in the protocol. The study protocol was discussed with the participating centers until consensus was reached.

Prior to opening inclusion, all participating centers will perform a dummy run in order to assess compliance with the protocols. Also during inclusion, quality assurance by central review will occur prospectively for the first 3 patients included at each participating center, and will occur retrospectively for all included patients thereafter.

\section{Discussion \\ Current status}

A total of 6 head and neck centers (or affiliated) will participate and include: the Radboudumc Nijmegen, University Medical Center Utrecht, VU University Medical Center Amsterdam, MAASTRO clinic Maastricht, Radiotherapiegroep Arnhem and Radiotherapeutisch Instituut Friesland.

The first patient was included in august 2016 and accrual is expected to continue for 4 years. 


\section{Abbreviations}

BTV: Biological target volume; CT: Computed tomography; CTC: Common toxicity criteria; CTV: Clinical target volume; EANM: European association of nuclear medicine; EARL: EANM research Ltd.; EBRT: External beam radiation therapy; EGFR: Epidermal growth factor receptor; EORTC: European organization for research and treatment of cancer; EQD2: Equivalent dose in 2 Gy fractions; FDG: Fluorodeoxyglucose (18F); FNAC: fine needle aspiration cytology; GRIX: Groningen radiation therapy induced xerostomia questionnaire; GTV: Gross tumor volume; IMRT: Intensity-modulated radiation therapy; MRI: Magnetic resonance imaging; PET: Positron emission tomography; PSS-HN: Performance status scale for head and neck cancer patients; PTV: Planning target volume; RTOG: Radiation therapy oncology group; SIB: Simultaneous-integrated boost; SUR: Standardized uptake ratio; SUV: Standardized uptake value; SWAL-QOL: Wwallowing quality of life questionnaire; US: Ultrasound; VMAT: Volumetric arc therapy

\section{Acknowledgements}

Not applicable.

\section{Funding}

The Dutch Cancer Society (KWF) provided financing for clinical trial data management and trial support (KUN 2015-7714). The subsidizing party is not involved in the collection, analysis, and interpretation of data nor in writing the manuscript.

\section{Availability of data and materials}

Data sharing is not applicable to this article as the current study is still open for inclusion of patients.

\section{Authors' contributions}

Study concept and design: JK and WO. Drafting of the trial protocol: SB. Critical revision of the trial protocol for important intellectual content: JK, TD, WO, MK, CT, CR, PD, FH, MV. Obtaining funding: SB, JK, TD, WO. Coordinating investigator: SB. Principal investigators at the various institutes: JK, PD, FH, MV, BK, OW. All authors read and approved the final manuscript.

\section{Competing interests}

The authors declare that they have no competing interests.

\section{Consent for publication}

Not applicable.

\section{Ethics approval and consent to participate}

This study will be conducted according to the ethical principles for medical research involving human subjects as stated in the Declaration of Helsinki and in the $\mathrm{ICH}$ Good Clinical Practice guidelines.

Written informed consent will be obtained from all participants included in the study prior to randomization. Patients will have the opportunity to ask any questions he/she might have and will have sufficient time to consider the implications of the study before deciding to participate.

Central ethical approval was provided for all 6 participating centers by the Committee on Research Involving Human Subjects of the region

Arnhem-Nijmegen and is registered under number NL46354.091.15.

\section{Publisher's Note}

Springer Nature remains neutral with regard to jurisdictional claims in published maps and institutional affiliations.

\footnotetext{
Author details

${ }^{1}$ Department of radiation oncology, Radboud University Medical Center, huispost 874, P.O. Box 9101, Nijmegen 6500, HB, The Netherlands. ${ }^{2}$ Department of radiation oncology, University Medical Center Utrecht, Utrecht, The Netherlands. ${ }^{3}$ Department of radiation oncology (MAASTRO), Research Institute GROW, Maastricht University, Maastricht, The Netherlands. ${ }^{4}$ Department of radiation oncology, VU University Medical Center, Amsterdam, The Netherlands. ${ }^{5}$ Department of radiation oncology, Radiotherapiegroep, Arnhem, The Netherlands. ${ }^{6}$ Department of radiation oncology, Radiotherapeutisch Instituut Friesland, Leeuwarden, The Netherlands. ${ }^{7}$ Department of nuclear medicine, Radboud University Medica Center, Nijmegen, The Netherlands. ${ }^{8}$ The Institute of Cancer Research and The Royal Marsden NHS Foundation Trust, London, UK.
}

Received: 11 April 2016 Accepted: 14 March 2017

Published online: 21 March 2017

\section{References}

1. Maccomb WS, Fletcher GH. Planned combination of surgery and radiation in treatment of advanced primary head and neck cancers. Am J Roentgenol Radium Ther Nucl Med. 1957;77(3):397-414.

2. de Bondt RB, Nelemans PJ, Hofman PA, Casselman JW, Kremer B, van Engelshoven JM, et al. Detection of lymph node metastases in head and neck cancer: a meta-analysis comparing US, USgFNAC, CT and MR imaging. Eur J Radiol. 2007:64(2):266-72.

3. Liao L, Lo WC, Hsu WL, Wang CT, Lai MS. Detection of cervical lymph node metastasis in head and neck cancer patients with clinically NO neck-a metaanalysis comparing different imaging modalities. BMC Cancer. 2012;12:236.

4. Suwinski $R$, Maciejewski $B$, Withers HR. Dose-response relationship for elective neck irradiation of head and neck cancer-facts and controversies. Neoplasma. 1998;45(2):107-12.

5. Withers HR, Peters $\sqcup$, Taylor JM. Dose-response relationship for radiation therapy of subclinical disease. Int J Radiat Oncol Biol Phys. 1995;31(2):353-9.

6. Zhou SM, Wong TZ, Marks LB. Using FDG-PET activity as a surrogate for tumor cell density and its effect on equivalent uniform dose calculation. Med Phys. 2004:31(9):2577-83.

7. Schinagl DA, Hoffmann AL, Vogel WW, van Dalen JA, Verstappen SM, Oyen WJ, et al. Can FDG-PET assist in radiotherapy target volume definition of metastatic lymph nodes in head-and-neck cancer? Radiother Oncol. 2009; 91(1):95-100.

8. Al-Mamgani A, van Rooij P, Tans L, Verduijn GM, Sewnaik A. Baatenburg de Jong RJ: A prospective evaluation of patient-reported quality-of-life after (chemo)radiation for oropharyngeal cancer: which patients are at risk of significant quality-of-life deterioration? Radiother Oncol. 2013;106(3):359-63.

9. Langendijk JA, Doornaert P, Rietveld DH, Verdonck-de Leeuw IM, Leemans CR, Slotman BJ. A predictive model for swallowing dysfunction after curative radiotherapy in head and neck cancer. Radiother Oncol. 2009:90(2):189-95.

10. Langendijk JA, Doornaert P, Verdonck-de Leeuw IM, Leemans CR, Aaronson NK, Slotman BJ. Impact of late treatment-related toxicity on quality of life among patients with head and neck cancer treated with radiotherapy. J Clin Oncol. 2008:26(22):3770-6.

11. Dijkema T, Raaijmakers CP, Ten Haken RK, Roesink JM, Braam PM, Houweling $A C$, et al. Parotid gland function after radiotherapy: the combined michigan and utrecht experience. Int J Radiat Oncol Biol Phys. 2010;78(2):449-53.

12. Terhaard CH, Dijkema T, Braam PM, Roesink JM, Raaijmakers CP. Abstract OC-0078: Sparing the contralateral submandibular gland in oropharyngeal cancer patients; dose-response analysis. Radiother Oncol. 2013; 106(Supplement 2):S30.

13. Eisbruch A, Kim HM, Feng FY, Lyden TH, Haxer MJ, Feng M, et al. ChemoIMRT of oropharyngeal cancer aiming to reduce dysphagia: swallowing organs late complication probabilities and dosimetric correlates. Int J Radiat Oncol Biol Phys. 2011;81(3):e93-9.

14. Levendag PC, Teguh DN, Voet P, van der Est H, Noever I, de Kruijf WJ, et al. Dysphagia disorders in patients with cancer of the oropharynx are significantly affected by the radiation therapy dose to the superior and middle constrictor muscle: a dose-effect relationship. Radiother Oncol. 2007;85(1):64-73.

15. Bakhshandeh M, Hashemi B, Mahdavi SR, Nikoofar A, Vasheghani M, Kazemnejad A. Normal tissue complication probability modeling of radiation-induced hypothyroidism after head-and-neck radiation therapy. Int J Radiat Oncol Biol Phys. 2013;85(2):514-21.

16. List MA, Ritter-Sterr C, Lansky SB. A performance status scale for head and neck cancer patients. Cancer. 1990:66(3):564-9.

17. van den Bosch S, Dijkema T, Verhoef LC, Zwijnenburg EM, Janssens GO, Kaanders JH. Patterns of Recurrence in Electively Irradiated Lymph Node Regions After Definitive Accelerated Intensity Modulated Radiation Therapy for Head and Neck Squamous Cell Carcinoma. Int J Radiat Oncol Biol Phys. 2016;94(4):766-74.

18. Boellaard R, Delgado-Bolton R, Oyen WJ, Giammarile F, Tatsch K, Eschner W, et al. FDG PET/CT: EANM procedure guidelines for tumour imaging: version 2.0. Eur J Nucl Med Mol Imaging. 2015;42(2):328-54.

19. van den Bosch S, Dijkema T, Hoebers F, Terhaard C, Kaanders J, Oyen W: Tumor to cervical spinal cord standardized uptake ratio improves the reproducibility of 18F-FDG-PET based tumor segmentation in head and neck squamous cell. Submitted to Journal. 2017 
20. Gregoire V, Ang K, Budach W, Grau C, Hamoir M, Langendijk JA, et al. Delineation of the neck node levels for head and neck tumors: a 2013 update. DAHANCA, EORTC, HKNPCSG, NCIC CTG, NCRI, RTOG, TROG consensus guidelines. Radiother Oncol. 2014;110(1):172-81.

21. Brouwer CL, Steenbakkers RJ, Bourhis J, Budach W, Grau C, Gregoire V, et al. $C T$-based delineation of organs at risk in the head and neck region: DAHANCA, EORTC, GORTEC, HKNPCSG, NCIC CTG, NCRI, NRG Oncology and TROG consensus guidelines. Radiother Oncol. 2015;117(1):83-90.

22. Santanam L, Hurkmans C, Mutic S, van Vliet-Vroegindeweij C, Brame S, Straube $W$, et al. Standardizing naming conventions in radiation oncology. Int J Radiat Oncol Biol Phys. 2012;83(4):1344-9.

23. Common Toxicity Criteria v2.0 (Revised March 23, 1998). http://ctep.cancer gov/protocolDevelopment/electronic_applications/ctc.htm

24. Cox JD, Stetz J, Pajak TF. Toxicity criteria of the Radiation Therapy Oncology Group (RTOG) and the European Organization for Research and Treatment of Cancer (EORTC). Int J Radiat Oncol Biol Phys. 1995;31(5):1341-6.

25. Dijkema T, Raaijmakers CP, Braam PM, Roesink JM, Monninkhof EM, Terhaard CH. Xerostomia: a day and night difference. Radiother Oncol. 2012;104(2):219-23.

26. Roesink JM, Moerland MA, Battermann JJ, Hordijk GJ, Terhaard CH. Quantitative dose-volume response analysis of changes in parotid gland function after radiotherapy in the head-and-neck region. Int J Radiat Oncol Biol Phys. 2001:51(4):938-46.

27. Patterson JM, McColl E, Carding PN, Kelly C, Wilson JA. Swallowing performance in patients with head and neck cancer: a simple clinical test. Oral Oncol. 2009;45(10):904-7.

28. Aaronson NK, Ahmedzai S, Bergman B, Bullinger M, Cull A, Duez NJ, et al. The European Organization for Research and Treatment of Cancer QLQ-C30: a quality-of-life instrument for use in international clinical trials in oncology. J Natl Cancer Inst. 1993;85(5):365-76.

29. Bjordal K, Ahlner-Elmqvist M, Tollesson E, Jensen AB, Razavi D, Maher EJ, et al. Development of a European Organization for Research and Treatment of Cancer (EORTC) questionnaire module to be used in quality of life assessments in head and neck cancer patients. EORTC Quality of Life Study Group. Acta Oncol. 1994;33(8):879-85

30. Beetz I, Burlage FR, Bijl HP, Hoegen-Chouvalova O, Christianen ME, Vissink A et al. The Groningen Radiotherapy-Induced Xerostomia questionnaire: development and validation of a new questionnaire. Radiother Oncol. 2010; 97(1):127-31.

31. McHorney CA, Robbins J, Lomax K, Rosenbek JC, Chignell K, Kramer AE, et al. The SWAL-QOL and SWAL-CARE outcomes tool for oropharyngeal dysphagia in adults: III. Documentation of reliability and validity. Dysphagia. 2002;17(2):97-114

32. Osoba D, Rodrigues G, Myles J, Zee B, Pater J. Interpreting the significance of changes in health-related quality-of-life scores. J Clin Oncol. 1998;16(1):139-44.

33. Pocock SJ. Group Sequential Methods in Design and Analysis of ClinicalTrials. Biometrika. 1977:64(2):191-200.

34. Thames HD, Withers HR, Peters LJ, Fletcher GH. Changes in early and late radiation responses with altered dose fractionation: Implications for dosesurvival relationships. Int J Radiation Oncol, Biol, Physics. 1982;8(2):219-26.

35. Withers HR, Taylor JM, Maciejewski B. The hazard of accelerated tumor clonogen repopulation during radiotherapy. Acta Oncologica. 1988;27(2): $131-46$

\section{Submit your next manuscript to BioMed Central and we will help you at every step:}

- We accept pre-submission inquiries

- Our selector tool helps you to find the most relevant journal

- We provide round the clock customer support

- Convenient online submission

- Thorough peer review

- Inclusion in PubMed and all major indexing services

- Maximum visibility for your research

Submit your manuscript at www.biomedcentral.com/submit

) Biomed Central 